\title{
Contribution to Fatigue Striation Phenomenon Analysis by Using Image Processing
}

\author{
Benoit Ruellan, Eric Robin, Jean-Benoit Le Cam, Isabelle Jeanneau, Frédéric Canévet, Gérard Mauvoisin, \\ and Didier Loison
}

\begin{abstract}
Since the use of energetic approaches for the prediction of the number at macro-crack initiation in elastomers, a special attention is paid on fatigue crack growth at the microscopic scale. In filled natural rubber, failure surfaces exhibit wrenchings and striations (Le Cam et al., Int J Fatigue 52:82-94, 2013). Both are assumed to be due to straininduced crystallization (SIC). Only four studies address fatigue striations (Le Cam et al., Int J Fatigue 52:82-94, 2013; Le Cam and Toussaint, Macromolecules 43:4708-4714, 2010; Flamm et al., Int J Fatigue 33:1189-1198, 2011; MuñozMejia, Dissertation, Université Claude Bernard, Lyon I, 2011), while they could provide information of importance to better understand how SIC enables natural rubber to resist the crack growth. As striations are similar to fringe patterns, this study aims at using a phase extraction algorithm from a single fringe pattern to analyse the striation morphology (Robin et al., Appl Opt 44:7261-7269, 2005; Takeda et al., J Opt Sot Am 72:156-160, 1982; Servin et al., Appl Opt 36(19):45404548, 1997; Robin et Valle, Appl Opt 43(22):4355-4361, 2004; Valle et al., Strain 46(2):175-183, 2008). This phase extraction methodology is split into three steps. The first one consists in extracting the wrapped phase without orientation. The second step is devoted to the determination of the fringe pattern orientation from a classic unwrapping algorithm. The third and last step consists in using an unwrapping algorithm (Zuo et al., Opt Lasers Eng 85:84-103, 2016; Menese et al., Appl Opt 44(7):1207-1215, 2005) and to compute the difference between the unwrapped phase processed and a plane in order to analyse the evolution of the striation morphology. This methodology has been applied to characterize the striation morphology observed at the failure surface of specimen tested under different fatigue loading conditions.
\end{abstract}

Keywords Fatigue striation $\cdot$ Phase demodulation $\cdot$ Single fringe pattern $\cdot$ Experimental mechanics $\cdot$ Rubber

\subsection{Introduction}

Fatigue striation patterns observed at the failure surface of samples submitted to fatigue loading conditions exhibit a similar morphology as a fringe pattern one, in the sense that they exhibit a periodic pattern [1-4]. This is illustrated in Fig. 17.1.

\author{
B. Ruellan $(\bowtie)$ \\ Univ Rennes, CNRS, IPR (Institute de Physique de Rennes)-UMR 6251, Rennes, France \\ LC-DRIME, Joint Research Laboratory, Cooper Standard-Institut de Physique UMR 6251, Rennes Cedex, France \\ Cooper Standard France, Rennes, France \\ e-mail: benoit.ruellan@cooperstandard.com \\ E. Robin · J.-B. Le Cam \\ Univ Rennes, CNRS, IPR (Institute de Physique de Rennes)-UMR 6251, Rennes, France \\ LC-DRIME, Joint Research Laboratory, Cooper Standard-Institut de Physique UMR 6251, Rennes Cedex, France \\ I. Jeanneau · F. Canévet \\ LC-DRIME, Joint Research Laboratory, Cooper Standard-Institut de Physique UMR 6251, Rennes Cedex, France \\ Cooper Standard France, Rennes, France \\ G. Mauvoisin \\ Laboratoire de Génie Civil et Génie Mécanique EA 3913, IUT-Université de Rennes 1, Rennes, France \\ D. Loison \\ Univ Rennes, CNRS, IPR (Institute de Physique de Rennes)-UMR 6251, Rennes, France
}




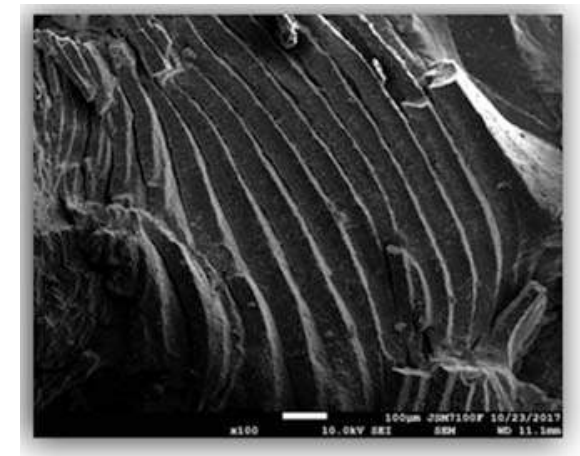

(a)

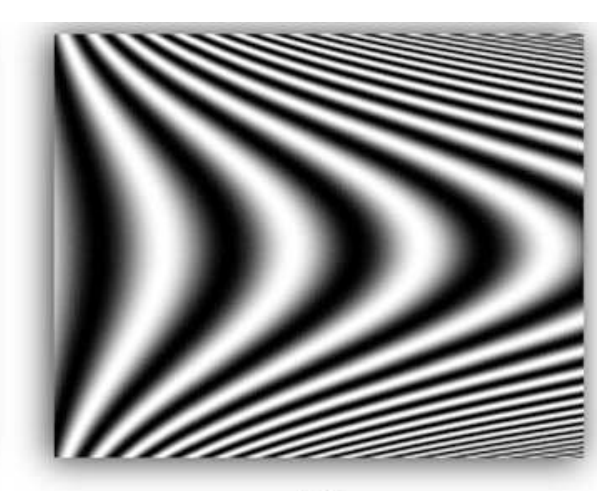

(b)

Fig. 17.1 (a) Striation pattern (b) fringes pattern

In an image (for instance that in Fig. 17.1a), the fringe pattern-like is issued from fatigue phenomenon and the corresponding phase cannot be shifted. This means that analysing fatigue striation as fringe pattern has to be carried out from a single image. Phase extraction from a single image was made possible by the development of analysis method such as the pMPC developed in [5-9]. The question addressed in the present paper is to know whether such analysis method can be directly applied to a SEM image of fatigue striation.

\subsubsection{Fringe Pattern Method Used for Analyzing Fatigue Striation: Modulation Phase Correlation (pMPC)}

The digital fringes pattern can be defined as follow:

$$
I(i, j)=A(i, j) \cos (\varphi(i, j))+B(i, j)
$$

where $A(i, j), B(i, j)$ and $\varphi(i, j)$ stand for the amplitude modulation, the background and the phase, respectively. The method is based on the measurement of the similarity degree between the fringe pattern studied and a virtual fringe pattern that is composed of parallel fringes. In other words, A, B and $\varphi$ are determined by minimizing the cost function:

$$
\Psi(A, B, \varphi)=\iint\left(\text { Val }_{\text {exp }}-(A \cos (\varphi)+B)\right)^{2}
$$

In practice, since the cost function describes in Eq. (17.2) is not unimodal, $\cos (\varphi)+B$ is replaced by its Maclaurin series expansion [5]. Indeed, we cannot ensure the unicity of A, B and $\varphi$ obtained from the minimization process, but this is the case with a polynomial form. The polynomial form writes as follows:

$$
\begin{aligned}
& A \cos \left(\frac{2 \pi}{p} X+\varphi\right)+B= \\
& A \cos (\varphi)+B-\frac{2 A \sin (\varphi) \pi}{p} X-\frac{2 A \cos (\varphi) \pi^{2}}{p^{2}} X^{2}+\frac{4 A \sin (\varphi) \pi^{3}}{3 p^{3}} X^{3}+O\left(X^{4}\right)
\end{aligned}
$$

where function $X=(x-\xi) \cos (\alpha)+(y-\gamma) \sin (\alpha)$ and $\alpha$ is the fringe inclination. In these conditions, variables optimizing the new cost function are the polynomial form's coefficients, in other words $\mathrm{C}_{0}, \mathrm{C}_{1}, \mathrm{C}_{2}$, and $\mathrm{C}_{3}$, and the cost function rewrites as follows:

$$
\psi_{p M P C}\left(c_{0}, c_{1}, c_{2}, c_{3}, \alpha\right)=\int_{N_{x y}}(I(x-\xi, y-\gamma)-P(\xi, \gamma))^{2} d \xi d \gamma
$$

with:

$$
P(\xi, \gamma)=c_{0}+c_{1} X+c_{2} X^{2}+c_{3} X^{3}
$$


and

$$
X=(x-\xi) \cos (\alpha)+(y-\gamma) \sin (\alpha)
$$

In this formulation, $\mathrm{A}, \mathrm{B}$ and $\varphi$ are not directly obtained from the optimization process but only $\mathrm{C}_{0}, \mathrm{C}_{1}, \mathrm{C}_{2}, \mathrm{C}_{3}$ and $\alpha . \varphi$ is then obtained by the analytical resolution of the equation system below:

$$
\left\{\begin{array}{c}
C_{0}=A \cos (\varphi)+B \\
C_{1}=-\frac{2 A \sin (\varphi) \pi}{p} \\
C_{2}=-\frac{2 A \cos (\varphi) \pi^{2}}{p^{2}} \\
C_{3}=\frac{4 A \sin (\varphi) \pi^{3}}{3 p^{3}}
\end{array}\right.
$$

and $\varphi$ chosen as:

$$
\varphi=\arctan \left(\frac{\pi C_{1}}{p C_{2}}\right)
$$

As the parameter $\alpha$ ranges between 0 and $\pi$ during the optimization process the maps giving the phase field unwrapped is not correctly oriented [8]. The correctly orientated phase field is obtained with the help of unwrapping process applied to $\alpha$ field. During this unwrapping process $[10,11]$ of $\alpha$ field, $\varphi$ field correctly orientated can be calculated by removing $\varphi$ by $(2 \pi-\varphi)$ when $\pi$ discontinuity is detected $[8]$.

\subsubsection{Applying pMPC to SEM Images}

In a first approach, a single striation pattern SEM image has been processed with the pMPC algorithm. The SEM used for the purpose of the study is a JSM JEOL 7100F. Results are presented in Fig. 17.2.
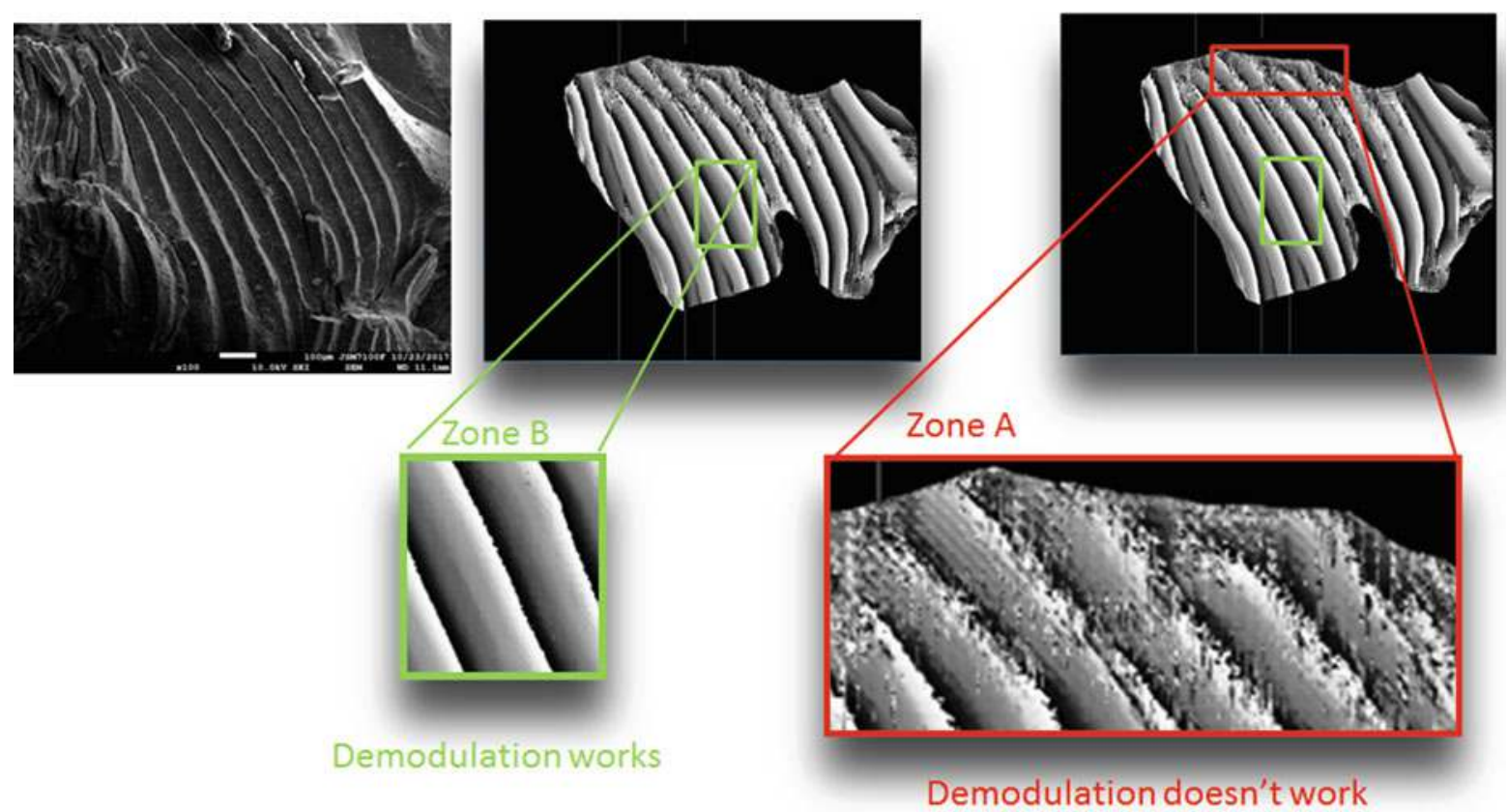

Fig. 17.2 Striation image directly analysed by pMPC 

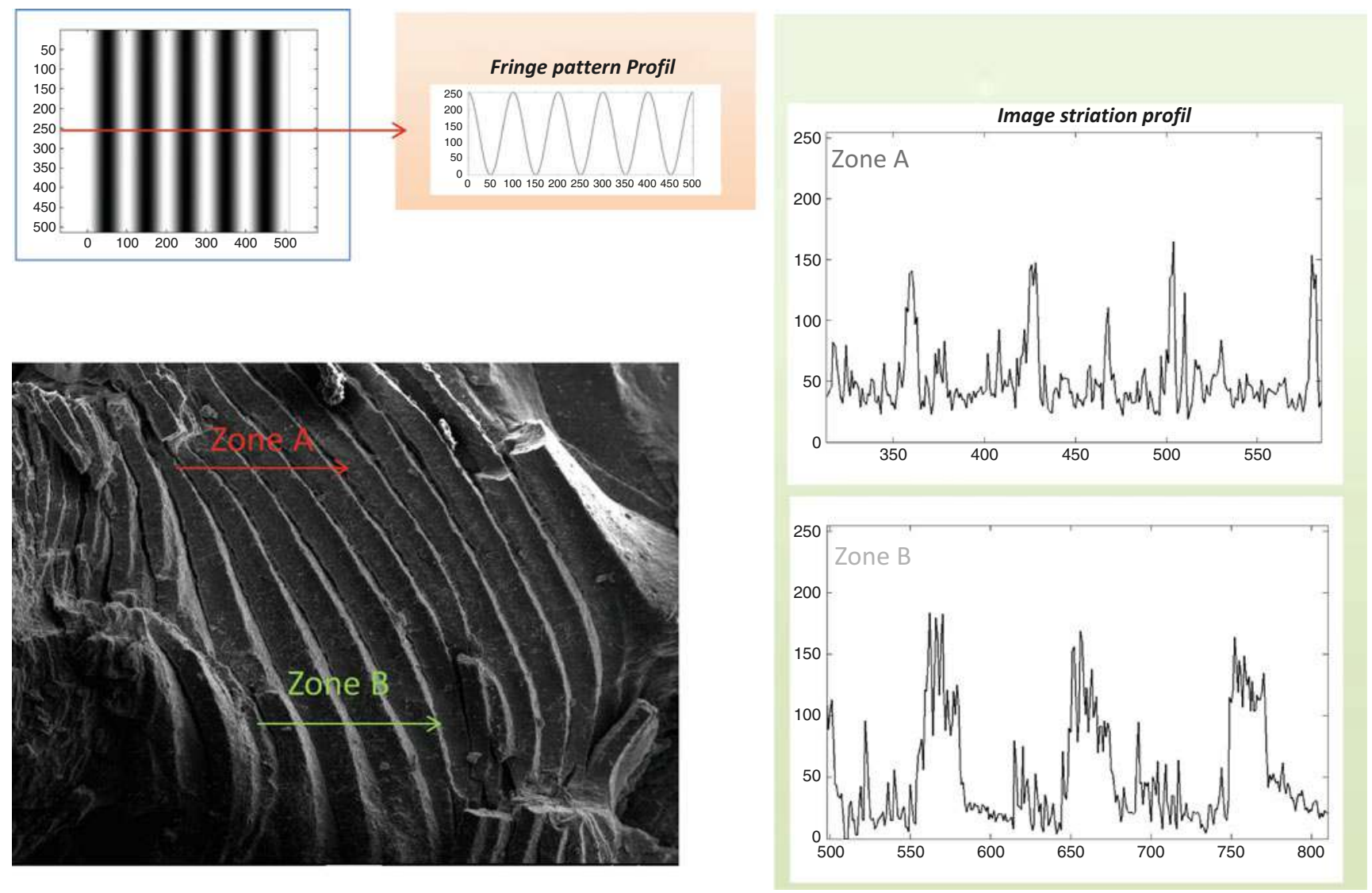

Fig. 17.3 Profiles of Zone A and B

Results show that the phase is correctly demodulated in zone B, but not in zone A, where the phase is strongly noisy or not correctly demodulated.

As shown by profiles in zone A and zone B in Fig. 17.3, the variation of the grey levels does not correspond to a fringe pattern. This explains why the phase is not extracted correctly in zone A (Fig. 17.2). Images, more especially the grey level dynamics, have therefore been processed in order to fit the grey level dynamics of a fringe pattern. Examining zone A profile shows that the signal is noisy but exhibits periodic peaks. These peaks should be larger and less noisy. The morphological modification of each peak is performed with the help of mathematical morphology in grey level. As morphology of profile becomes a square periodic signal with this type of morphological operation, we propose to use the edge effect of median filter in order to remove this square effect.

Note that such a filter reduces the noise. The kernel size has to be lower than a quarter of the signal period [12]. Applying this filter leads to a demodulated field where noisy zones disappear. Results obtained with the phase extraction algorithm are presented in Fig. 17.4.

In order to highlight the variation in the striation pitch, a numerical plane is subtracted from the continuous unwrapped phase field. 

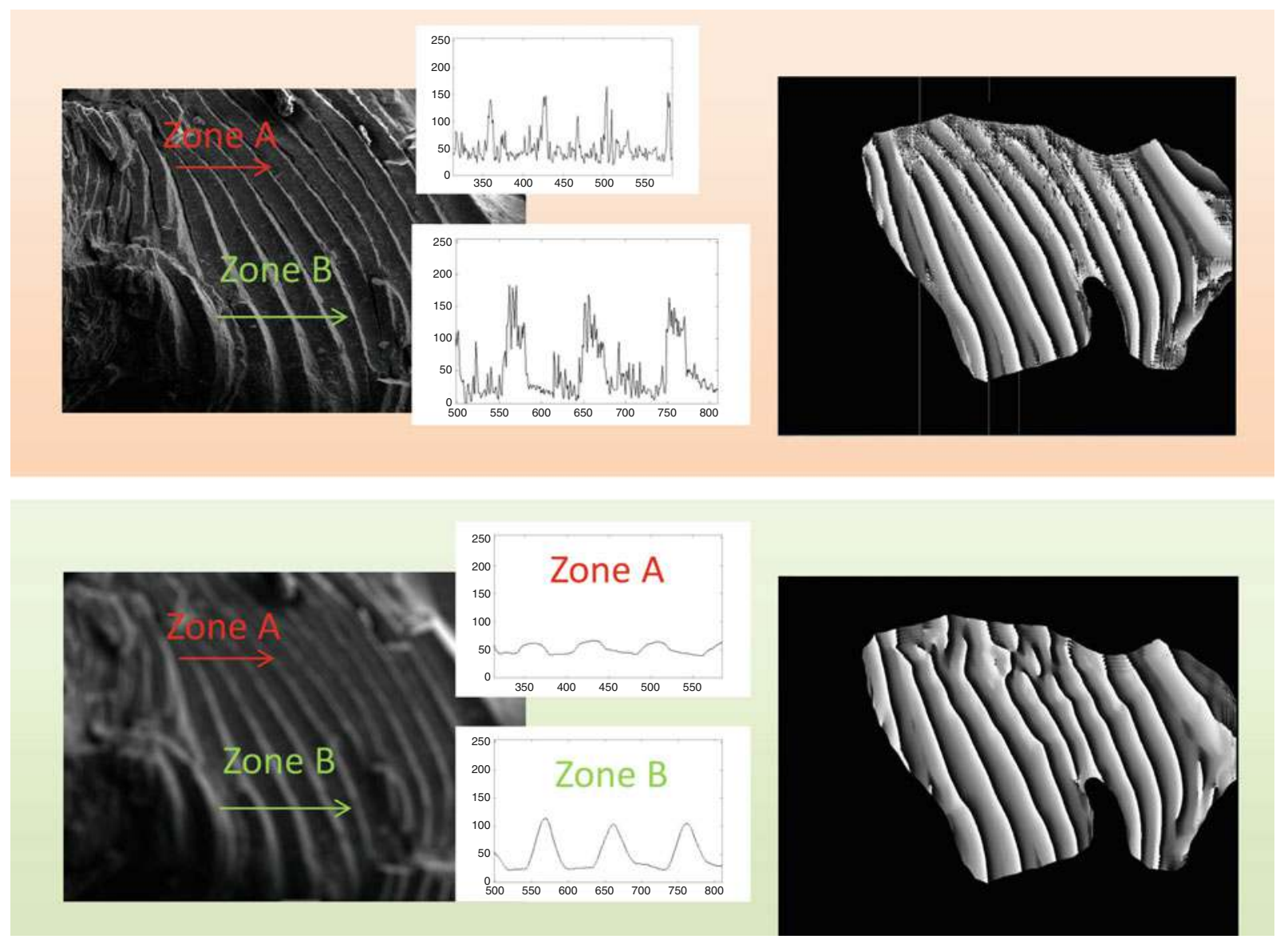

Fig. 17.4 Striation pattern processed for phase demodulation

\subsection{Results}

The methodology previously described has been applied on six different SEM images exhibiting fatigue striations. These striations have been obtained from different loading conditions applied to Diabolo samples (see [13]). Figures 17.5 and 17.6 present results obtained, which will be more precisely detailed and discussed during the presentation.

\subsection{Conclusion and Perspectives}

In this paper, a non-destructive technique is proposed to study fatigue striation based on a phase extraction algorithm. Results presented remain qualitative and not quantitative. Indeed, SEM images are affected by perspective effects (the different zones at the surface observed are not in the same plane), which induces the detection of apparent striation pitch variation by the algorithm. In order to access quantitative data, the striation topology is required. It can be characterized by different metrological devices, but the small striation height and the large out-of-plane zones makes difficult the measurement. This is why topology characterization of fatigue striation with SEM is currently under study in our lab. 

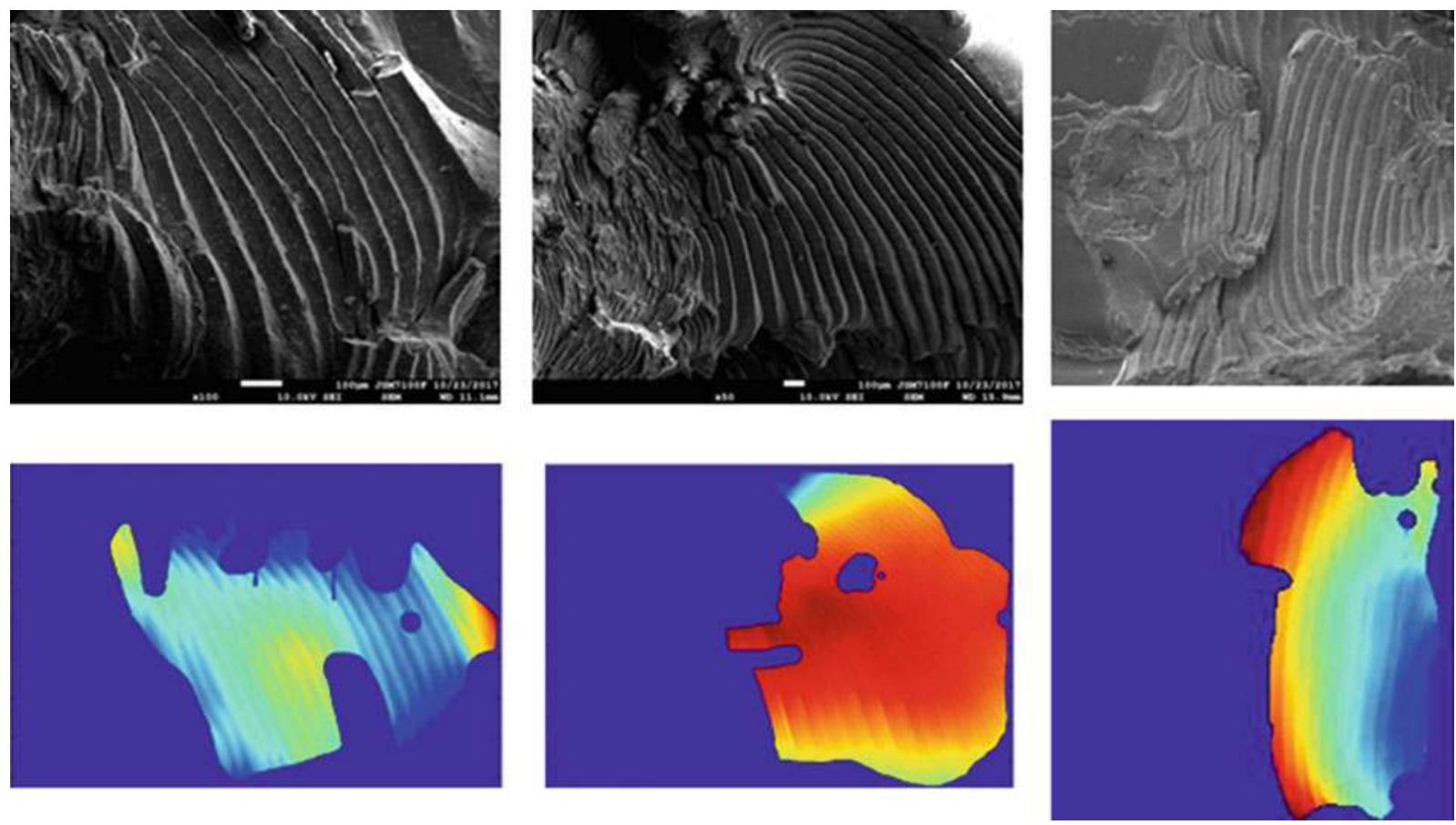

Fig. 17.5 SEM images analysis by phase demodulation image processing
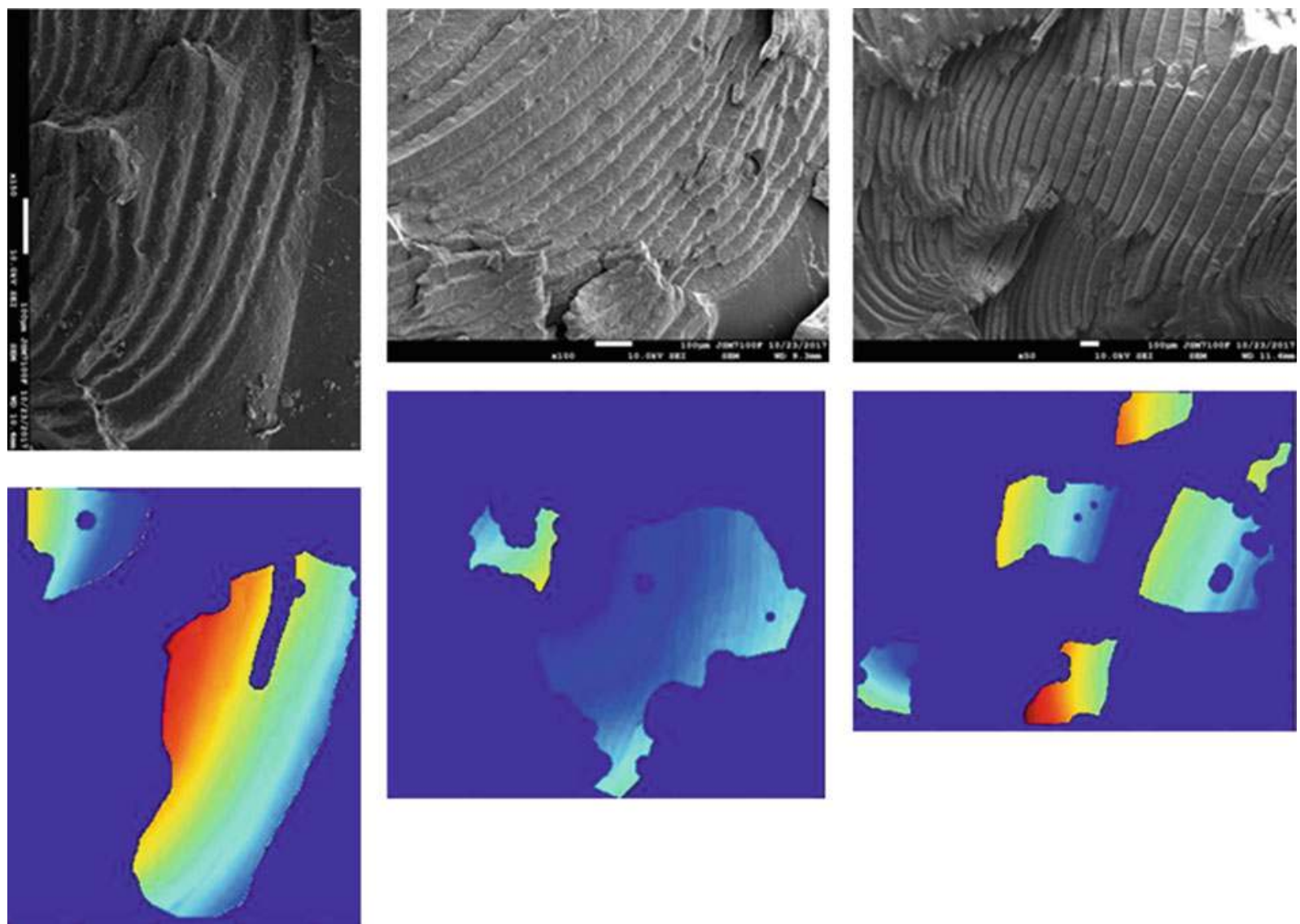

Fig. 17.6 SEM images analysis by phase demodulation image processing 


\section{References}

1. Le Cam, J.-B., Huneau, B., Verron, E.: Fatigue damage in carbon black filled natural rubber under uni- and multiaxial loading conditions. Int. J. Fatigue. 52, 82-94 (2013)

2. Le Cam, J.-B., Toussaint, E.: The mechanism of fatigue crack growth in rubbers under severe loading: the effect of stress-induced crystallization. Macromolecules. 43, 4708-4714 (2010)

3. Flamm, M., Spreckels, J., Steinweger, T., Weltin, U.: Effects of very high loads on fatigue life of NR elastomer materials. Int. J. Fatigue. 33, 1189-1198 (2011)

4. Muñoz-Mejia, L.: Étude expérimentale des mécanismes d'endommagement par fatigue dans les élastomères renforcés. Ph.D. dissertation, Université Claude Bernard, Lyon I (2011)

5. Robin, E., Valle, V., Brémand, F.: Phase demodulation method from a single fringe pattern based on correlation with a polynomial form. Appl. Opt. 44, 7261-7269 (2005)

6. Takeda, M., Hideki, I., Kobayashi, S.: Fourier-transform method of fringe pattern analysis for computer based topography and interferometry. J. Opt. Sot. Am. 72, 156-160 (1982)

7. Servin, M., Marroquin, J.-L., Cuevas, F.-J.: Demodulation of a single interferogram by use of a two-dimensional regularized phase-tracking technique. Appl. Opt. 36(19), 4540-4548 (1997)

8. Robin, E., Valle, V.: Phase demodulation from a single fringe pattern based on a correlation technique. Appl. Opt. 43(22), 4355-4361 (2004)

9. Valle, V., Robin, E., Bremand, F.: Analysis of digital speckle-pattern interferometry fringe patterns issued from transitory mechanical loading. Strain. 46(2), 175-183 (2008)

10. Zuo, C., Huang, L., Zhang, M., Chen, Q., Asundi, A.: Temporal phase unwrapping algorithms for fringe projection profilometry: a comparative review. Opt. Lasers Eng. 85, 84-103 (2016)

11. Menese, J., Gharbi, T., Humbert, P.: Phase-unwrapping algorithm for images with high noise content based on a local histogram. Appl. Opt. 44(7), 1207-1215 (2005)

12. Corvec, G., Robin, E., Le Cam, J.-B., Sangleboeuf, J.-C., Lucas, P.: Improving spatio-temporal resolution of infrared images to detect thermal activity of defect at the surface of inorganic glass. Infrared Phys. Technol. 77, 193-202 (2016)

13. Ruellan, B., Le Cam, J.-B., Robin, E., Jeanneau, I., Canévet, F., Mortier, F.: Influence of the temperature on lifetime reinforcement of a filled NR. In: Conference and Exposition on Experimental and Applied Mechanics SEM 2018, Greenville, 4-7 June 2018 